\title{
Sur une propriété des ensembles $(A)$.
}

Par

\author{
W. Sierpiński (Varsovie).
}

Nous avons démontré avec M. Lusin en 1923 ) que tout ensemble ( $A$ ) de $M$. Souslin est une somme de $\boldsymbol{N}_{1}$ ensembles mesurables $B{ }^{2}$ ). Le but de cette note est de donner une démonstration plus simple et directe de cette propriété et 'd'en donner une généralisation.

1. Supposons qu'à tout système fini de nombres naturels $n_{1}, n_{2}, \ldots$, $n_{k}$ corresponde un intervalle $\delta_{n_{1}, n_{2}, \ldots, n_{k}}$; on dit qu'on a un système déterminant $S\left\{\delta_{n_{1}, n_{2}, \ldots, n_{k}}\right\}$. On appelle noyau du système $S$ l'ensemble-somme de tous les produits infiñis

$$
\delta_{n_{1}} \cdot \delta_{n_{1}, w_{2}} \cdot \delta_{n_{1}, n_{2}, n_{8}} \ldots,
$$

la sommation s'étendant à toutes les suites ínfinies de nombres na. turels $n_{1}, n_{2}, n_{3}, \ldots$.

On appelle ensemble (A) (linéaire) tout ensemble qui est noyau d'un système déterminant.

Soit $E$ un ensemble $(A)$ donné, noyau du système $S\left\{\delta_{n_{1}, n_{2}, \ldots, n_{k}}\right\}$ : Posons, pour tout système fini de nombres náturels $n_{1}, n_{\mathbf{2}}, \ldots, n_{k}$ :

$$
\delta_{n_{1}, n_{2}, \ldots, n_{k}}^{0}=\delta_{n_{1}, n_{3}, \ldots, n_{k}}
$$

1) N. Lusin et W: Sierpinski: Sur un ensemble non mesurable B. Journal de Mathómatiques, t. II (1923), p. 52.

2) La démonstration d'une propriété analogue des ensembles complémentaires aux ensembles $(A)$ se troure déjà dans le mémoire de N. Lusin et W. Sierpinski: Sur quelques propriétés des ensembles (A). Bull. Acad, Cracovie 1918,
p. 39. 


$$
\delta_{n_{1}, n_{2}, \ldots, n_{k}}^{\alpha+1}=\delta_{n_{1}, n_{2}, \ldots, n_{k}}^{\alpha} \sum_{n=1}^{\infty} \delta_{n_{1}, n_{2}, \ldots, n_{k_{k}}, n}^{\alpha}
$$

pour tout nombre ordinal $a<\Omega$, et

$$
\delta_{n_{1}, n_{2}, \ldots, n_{k}}^{\alpha}=\prod_{\xi<\alpha} \delta_{\xi_{k}, n_{3}, \ldots, n_{k}}^{\xi}
$$

pour tout nombre ordinal $\alpha$ de seconde espèce, le produit $I I$ s'étendant à tous les nombres ordinaux $\xi<\alpha$.

Les ensembles $\delta_{n_{1} n_{2}, \ldots, n_{k}}^{\alpha}$, définies ainsi par linduction transfinie, sont évidemment mesurables (B) pour tout système fini d'indices $n_{1}, n_{2}, \ldots, n_{k}$ et tout nombre ordinal $\alpha<Q$.

D'après (2) et (3) on trouve tout de suite par l'induction transfinie:

(4)

Posons

$$
\delta_{n_{1}, n_{2}, \ldots, n_{k}}^{\alpha} \subset \delta_{n_{1}, n_{2}, \ldots, n_{k}}^{\beta} . \text { pour } \alpha \geqslant \beta .
$$

$$
S^{\alpha}=\sum_{n=1}^{\infty} \delta_{n}^{\alpha}
$$

et

$$
T^{\alpha}=\sum_{\left(n_{1}, n_{2}, \ldots, n_{k}\right)}\left(\delta_{m_{1}, n_{2}, \ldots, n_{k}}^{\alpha}-\delta_{n_{1}, n_{2}, \ldots, n_{k}}^{\alpha+1}\right),
$$

la sommation dans (6) s'étendant à tous les systèmes finis de nombres naturels $n_{1}, n_{2}, \ldots, n_{k}$.

Les ensembles $S^{\alpha}$ et $T^{\alpha}$, ainsi que leurs différences $S^{\alpha}-T^{\alpha}$, seront évidemment tous mesurables (B) pour $\alpha<Q$.

Nous prouverons que

$$
E=\sum_{\alpha<\Omega}\left(S^{\alpha}-T^{\alpha}\right)
$$

la somination s'étendant à tous les nombres ordinaux $a<Q$.

Soit $\alpha$ un nombre ordinal donné $<Q$ et soit $x$ un élément de l'ensemble $S^{\alpha}-T^{\alpha}$. Nous avons donc

$$
x \varepsilon S^{\alpha}
$$

et $x$ non $\varepsilon T^{\alpha}$.

De (8) et de (5) résulte qu'il existe un nombre naturẹl $m_{1}$, tel que $x \varepsilon \delta_{m_{1}}^{\alpha}$. Or, de (9) et de (6) résulte que $x$ non $\varepsilon\left(\delta_{m_{1}}^{\alpha}-\delta_{m_{1}}^{\alpha+1}\right)$ 
(puisque $\delta_{m_{1}}^{\alpha}-\delta_{m_{1}}^{\alpha+1}$ est un des termes de la somme (6)): d'après $x \varepsilon \delta_{m_{1}}^{\alpha}$ nous trouvons done $x \varepsilon \delta_{m_{1}}^{\alpha+1}$. Or, d'après (2):

$$
\delta_{m_{1}}^{\alpha+1}=\delta_{m_{1}}^{\alpha} \sum_{n=1}^{\infty} \delta_{m_{1}, n}^{\alpha}
$$

et de $x \varepsilon \delta_{m_{1}}^{\alpha+1}$ résulte qu'il existe un indice $m_{2}$, tel que $x \varepsilon \delta_{m_{1}, m_{2}}^{\alpha}$ D'après (9) et (6) nous avons $x$ non $\varepsilon\left(\delta_{m_{1}, m_{2}}^{\alpha}-\delta_{m_{1}, m_{2}}^{\alpha+1}\right)$ : la formule $x \varepsilon \delta_{m_{1} m_{2}}^{\alpha}$ donne done $x \varepsilon \delta_{m_{1}, m_{2}}^{\alpha+1}$. Or, d'après (2)

$$
\delta_{m_{1}, m_{2}}^{\alpha+1} \subset \sum_{n=1}^{\infty} \delta_{m_{1}, m_{2}, n}^{\alpha}
$$

et de $x \varepsilon \delta_{m_{1}, m_{2}}^{\alpha+1}$ résulte l'existence d'un indice $m_{3}$, tel que $x \varepsilon \delta_{m_{1}, m_{2}, m_{\mathrm{B}}}^{\alpha}$. En raisonnant ainsi de suité nous obtenons une suite infinie d'indices $m_{1}, m_{2}, m_{3}, \ldots$, telle que

$$
x \varepsilon \delta_{m_{1}, m_{2}, \ldots, m_{k}}^{\alpha}, \text { pour } k=1,2,3, \ldots
$$

D'après (1) et (4) (pour $\beta=0$ ) il en résulte que

$$
x \varepsilon \delta_{m_{1}, m_{2}, \ldots, m_{k}} \text { pour } k=1,2,3, \ldots \text {, }
$$

donc, d'aprés la définition du noyan $E$ du système $S\left\{\delta_{n_{1}, n_{2}, \ldots, n_{k}}\right\}$; que $x \varepsilon E$.

Nous avons ainsi démontré que $\left(\Phi^{\alpha}-T^{\alpha}\right) C_{-} E$ pour tout nombre ordinal $\alpha<\Omega$ : il en résulte que

$$
\sum_{\alpha<\Omega}\left(S^{\alpha}-T^{\alpha}\right) \subset E
$$

Or, soit $x$ un élément de l'ensemble $E$. Il existe donc une suite infinie d'indices $m_{1}, m_{2}, m_{3}, \ldots$, telle que

$$
x \varepsilon \delta_{m_{1}, m_{2}, \ldots, m_{k}} \quad(k=1,2,3, \ldots)
$$

Je dis que

$$
x \varepsilon \delta_{m_{1}, m_{2}, \ldots, m_{k}}^{\alpha} \quad(k=1,2,3, \ldots)
$$

pour tout nombre ordinal $\alpha<\Omega$. D'après (11) et (1) la formule (12) est vraie pour $\alpha=0$. Or, soit $\beta$. un nombre ordinal $<\Omega$ et supposons que la formule (12) est vraie pour tout nombre ordinal $\alpha<\beta$. Si $\beta$ est un nombre de seconde espèce, il en résulte, d'après (3), que la formule (12) est vraie pour le nombre $\beta$. Si $\beta$ est un 
nombre de première espèce, nous pouvons poser $\beta=\alpha+1$, où $\alpha<\beta$, et la formule (2) donne (pour tiut nombre naturel $k$ )

$$
\delta_{m_{1}, m_{2}, \ldots, m_{k}}^{\beta}=\delta_{m_{1}, n_{2}, \ldots, m_{k}}^{\alpha} \sum_{n=1}^{\infty} \delta_{m_{1}, m_{2}, \ldots, m_{k^{\prime}, n}}^{\alpha} \supset \delta_{m_{1}, m_{2}, \ldots, m_{k}}^{\alpha} \delta_{m_{1}, \ldots, m_{k^{\prime}} m_{k+1}}^{\alpha}
$$

donc $x \varepsilon \delta_{m_{n_{1}, m_{2}, \ldots, m_{k}}^{\beta}}^{\beta}$ puisque, d'après (12) on a $x \varepsilon \delta_{m_{1}, m_{2}, \ldots, n_{k}}^{\alpha}$ et $x \varepsilon \delta_{m_{1}, m_{1}, \ldots, m_{k}, m_{k+1}}^{c}$. La formule (12) est ainsi établie par linduction transfinie pour tout nombre ordinal $\alpha<\Omega$.

En particulier, il résulte de (12) que $x \varepsilon \dot{\delta}_{m_{1}}^{\alpha}$ pour $\alpha<\Omega$, donc, d'après (5), que $x \varepsilon S^{a}$ pour $\alpha<Q$. Nous avons ainsi démontré que $E \subset S^{\alpha}$ pour $\alpha<\Omega$, done que

$$
E \subset \prod_{\alpha<Q} S^{\alpha}
$$

Je dis, maintenant, que

$$
\prod_{\alpha<0} T^{\alpha}=0
$$

Admettons, en effet, que la formule (14) n'est pas vraie. Il existe done un nombre $x$ tel que

$$
x \varepsilon T^{\alpha} \text { pour } \alpha<Q .
$$

De (15) et (6) résulte qu'il existe pour tout nombre ordinal $\alpha<\Omega$ au moins un système d'indices $n_{1}, n_{2}, \ldots, n_{k}$ (dépendant de $\alpha$ ), tel que

$$
x \varepsilon\left(\delta_{n_{1}, n_{1}, . ., n_{k}}^{a}-\delta_{n_{1}, n_{2}, \ldots, n_{k}}^{\alpha+1}\right) .
$$

Or, l'ensemble de tous les systèmes finis d'indices $\left(n_{1}, n_{2} \ldots, n_{k}\right)$ étant dénombrable et l'ensemble de tous les nombres ordinaux $\alpha<Q$ étant non dénombrable, il en résulte tout de suite l'existence d'un système d'indices $p_{1}, p_{2}, \ldots, p_{r}$ (indépendant de $\alpha$ ) et de deux nombres ordinanx $\xi<\Omega$ et $\eta<\xi$, tels que

$$
x \varepsilon\left(\delta_{p_{1}, p_{2}, \ldots, p_{r}}^{\xi}-\delta_{p_{1}, p_{2}, \ldots, p_{r}}^{\xi+1}\right)
$$

et

$$
x \varepsilon\left(\delta \eta_{p_{1}, p_{2}, \ldots, p_{r}}-\delta \eta_{p_{1}, p_{2}, \ldots, p_{r}}^{+1}\right)
$$

De (16) et (17) résulte que

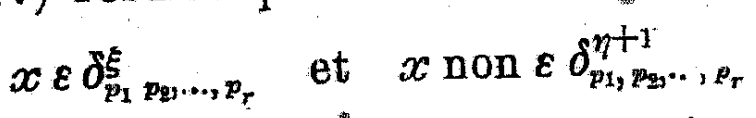

ce qui est incompatible avec (4), puisque (d'après $\eta<\xi) \eta+1 \leq \xi$. La formule (14) est ainsi établie. 
Soit maintenant $x$ un élément de l'ensemble $E . D^{c}$ après (14) il existe un nombre ordinal $\alpha<\Omega$ tel que $x$ non $\varepsilon T^{a}$. Or, d'après (13), nous avons $x \in S^{\alpha}$. Done $x \varepsilon\left(S^{\alpha}-T^{\alpha}\right)$. Nous avons ainsi démontré que si $x \varepsilon E$, il existe un nombre ordinal $\alpha<\Omega$, tel que $x \varepsilon\left(S^{a}-T^{\alpha}\right)$. Il en résulte que

$$
E \subset \sum_{\alpha=\Omega}\left(S^{\alpha}-T^{\alpha}\right)
$$

Les formules (10) et (18) donnent la formule (7), c. q. f. d.

Observons encore que les formules (7), (13) et (14) donnent tout de suite la formule

$$
E=\prod_{\alpha<\Omega} S^{\alpha} .
$$

En effet, soit $x$ un élément de l'ensemble $P=\prod_{\alpha \triangleleft \Omega} S^{\alpha}$. D'après (14), il existe un nombre ordinal $\alpha<\Omega$, tel que $x$ non $\varepsilon T^{\alpha}$. Or, de $x \varepsilon P$ résulte que $x \in S^{\alpha}:$ nous avons donc $x \varepsilon\left(S^{\alpha}-T^{\alpha}\right)$, donc, d'après (7), $x \dot{\varepsilon} E$. Nous trouvons ainsi $P C E$ et la formule (13) donne la formule (19). Il en résulte que tout ensemble complémentaire d'un ensemble (A) est une somme de $\aleph_{1}$ ensembles mesurables $B$.

2. Nous prouverons maintenant la proposition suivante qui peut être regardée comme une généralisation des propriétés démontrées dans le $\S 1$ :

Tout ensemble qu'on obtient en partant des ensembles (A) et ent effectuant dans un ordre quelconque un nombre fini ou une intinité dénombrable d'additions, de soustractions et de multiplications d'ensembles est une somme de $\aleph_{1}$ ensembles mesurables $B$.

$F^{\prime}$ étant une famille donnée quelconque d'ensembles et $E_{n_{1}, n_{2}, \ldots, n_{k}}$ étant des ensembles de la famille $F$, on dit que le noyan du système $S\left\{E_{n_{1}, n_{g}, \ldots, n_{k}}\right\}$ est un résultat de l'opération $A$ effectuée sur les ensembles de la famille $F$.

$K_{1}$ étant la famille de tous les ensembles linéaires complémentaires aux ensembles (A), désignons par $K_{2}$ la famille de tous les ensembles résultants de l'opération $A$ effectuée sur les ensembles de la famille $K_{1}$, et désignons par $K_{\mathbf{3}}$ la famille de tous les ensémbles linéaires dont les complémentaires appartiennent à $K_{\mathrm{q}}$.

Soit $H$ un ensemble donné quelconque de la famille $K_{8}$. L'ensemble $E=C H$ appartient donc à $K_{2}$ et parsuite il est noyau d'un 
système $S\left\{E_{n_{1}, n_{2}, \ldots, n_{k}}\right\}$, où les ensembles $E_{n_{1}, n_{2}, \ldots, n_{k}}$ sont complémentaires aux ensembles $(A)$.

Posons $\delta_{n_{1}, n_{0}, \ldots, n_{k}}=E_{n_{1}, n_{1}, \ldots, n_{k}}$ et définissons les ensembles $\delta_{n_{1}, n_{2}, \ldots, n_{k}}^{\alpha}$ et $S^{\alpha}$ par les formules (1), (2), (3) et (5). Nous aurons la formule (19) (puisque la démonstration de cette formule, donné dans le $\$ 1$, n'utilisait pas l'hypothèse que les ensembles $\delta_{n_{1}, n_{3}, \ldots, n_{k}}$ sont des intervalles), done aussi la formule

$$
C E=\sum_{\alpha<\Omega} C S^{\alpha}
$$

Or, les ensembles $C \delta_{n_{1}, n_{3} \ldots, n_{k}}$ étant des ensembles $(A)$ et la somme et le produit d'une infinité dénombrable d'ensembles (A) étant, comme on sait, un ensemble $(A)$, il résulte sans peine des formules (1), (2), (3) et (5) (et des formules de de Morgan) que les ensembles $C S^{\alpha}$ sont de ensembles (A). Donc, d'après (20), $H=C E$ est une somme de $\aleph_{1}$ ensembles $(A)$. Or, tout ensemble $(A)$ étant, comme nous l'avons démontré dans le $\$ 1$, une somme de $\aleph_{1}$ ensembles mesurables $B$, il en résulte tout de suite (d'après la formule $s_{1}^{2}=s_{1}$ ) que $H$ est une somme de $s_{1}$ ensembles mesurables $B$.

Nous avons ainsi démontré que tout ensemble de la famille $K_{3}$ est une somme de $s_{1}$ ensembles mesurables $B$.

Or, désignons par $K_{4}$ la plus petite classe $K$ d'ensembles satisfaisant aux quatre conditions suivantes:

1) Tout ensemble (A) linéaire appartient à $K$.

2) Tout ensemble linéaire complémentaire à un ensemble (A) appartient à $K$.

3) Si les ensembles $E_{1}, F_{q}, E_{9}, \ldots$ appartiennent à $K$, leur somme $E_{1}+E_{2}+E_{3}+\ldots$ appartient à $K$.

4) Si les ensembles $E_{1}, E_{2}, E_{3}, \ldots$ appartiennent à $K$, leur produit

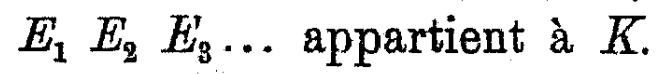

Je dis que $K_{4} \subset K_{3}$.

La classe $K_{1}$ étant, par définition, la partie commune (produit) de tontes les classes $K$ d'ensembles linéaires satisfasant aux conditions 1) -4), il suffira évidemment de démontrer que $K_{\mathrm{g}}$ est une des classes $K$ satisfaisant aux conditions 1)-4). 
Soit $E$ un ensemble (A) linéaire. L'ensemble $C E$ peut être évidemment régardé comme résultat de l'opération $A$ effectuée sur les ensembles qui. sont tous égaux à $C E$ : done $C E$ appartient à la classe $K_{\mathbf{q}}$ et pursuite $E$ appartient à $K_{\mathbf{g}}$. La classe $K=K_{\mathfrak{3}}$ jouit donc de la propriété 1).

Or, tout ensemble $(A)$ linéaire étant un résultat de l'opération $A$ effectuée sur des intervalles, donc sur les ensembles dont les complémentaires sont des ensembles $(A)$, on voit que tout ensemble $(A)$ linéaire appartient à la classe $K_{\mathrm{a}}$ et parsuite tout ensemble linéaire complémentaire à un ensemble (A) appartient à la classe $K_{\mathfrak{z}}$. La classe $K=K_{3}$ jouit done de la propriété 2).

On peut démontrer que.si $E_{1}, E_{2}, E_{8}, \ldots$ est une suite infinie d'ensembles dont chacun est résultat de l'operation $A$ effectuée sur les ensembles d'une famille $F$, il en est de même de leur somme $E_{1}+E_{2}+E_{3}+\ldots$ et de leur produit $E_{1} E_{2} E_{3} \ldots$ (La démonstration est tout à fait analogue à la démonstration qu'une somme et qu'un produit d'une infinité dénombrable d'ensembles (A) sont des ensembles (A)). Il en résulte que la classe $K=K_{2}$, et parsuite aussi la elasse $K=K_{3}$ jouit des propriétés 3) et 4 ) (Puisque de 3), resp. 4) pour $K_{2}$ résulte 4 ), resp. 3) pour $K_{8}$ ).

Nous avons ainsi démontré que $K_{4} \subset K_{8}$.

Or, je dis que si $E$ est un ensemble de $K_{4}, C E$ l'est aussi. Pour le prouver désignons par $K_{5}$ la classe de tous les ensembles linéaires dont le complémentaires appartiennent à $K_{4}$. La classe $K=K_{4}$ jouissant des propriétés 1) -4), on voit sans peine que la classe $K=K_{5}$ jouit aussi de ces propriétés. Done, $K_{5}$ étant une des classes $K$ jouissant des propriétés 1)-4), nous trouvons $K_{4} \subset K_{5}$. Done, si $E$ appartient à $K_{4}, E$ appartient aussi à $K_{5}$, et il résulte de la définition de lá classe $K_{5}$ que $C E$ appartient à $K_{4}$.

Il en résulte de la formule $E_{1}-E_{2}=E_{1} . C E_{2}$ et de la pro: priété 4) de la classe $K=K_{4}$ que cette classe jouit encore de la propriété 5) suivante:

5) La différence de deux ensembles appartenant à $K$ appartient à $K$.

Des propriétés 1), 3), 4) et 5) de la classe $K=K_{4}$ résulte que tout ensemble qu'on obtient en effectuant dans un ordre quelconque un nombre fini ou une infinité dénombrable d'additions, de soustractions et de multiplications d'ensembles à partir de ensembles $(A)$, appartient à $K_{4}$, donc, d'après $K_{4} \subset K_{3}$, aussi à $K_{3}$, et parsuite est une somme de $\kappa_{1}$ ensembles mesurables $B$. 
Notre théorème est ainsi démontré.

La proposition démontrée est encore susçeptible d'une généralisation. On voit notamment sans peine que $K_{4} \subset K_{2}$ et on pourrait démontrer que tout ensemble de $K_{2}$ est une projection d'un ensemble plan complémentaire d'un ensemble $(A)$; or, toute projection d'un ensemble plan complémentaire d'un ensemble $(A)$ est une somme de $\kappa_{1}$ ensemble mesurables $B{ }^{1}$ ).

D'après une remarque de $\mathrm{M}$. $\mathrm{Kuratowski}$ notre théorème admet encore une géuéralisation dans un autre sens: on pourrait notamment démontrer que tout ensemble qu'on obtient en partant des intervalles et en effectuant un nombre fini de fois les opérations $A$ et $C$ (complémentaire) est une somme de $\aleph_{1}$ ensembles mesurables $\dot{B}$ (Or, on ne saít pas si ce théorème subsiste pour une infinité dénombrable d'opérations $A$ et $($ ):

1) Cf. Fund. Muth. t. VII, p. 240. 